\title{
Planned Notification Study Site Accrual Threshold Percent
}

National Cancer Institute

\section{Source}

National Cancer Institute. Planned Notification Study Site Accrual Threshold Percent. NCI

Thesaurus. Code C94014.

An integer specifying a percentage of target accrual at a particular study site, which when reached triggers a notification. 